\title{
Periostin Expression and Its Roles in Benign and Malignant Thyroid Nodules: an Immunohistochemical Study of 105 Cases
}

Kimihide Kusafuka ( $\boldsymbol{\nabla}$ k-kusafuka@i.shizuoka-pho.jp )

Shizuoka General Hospital https://orcid.org/0000-0003-3482-7447

Masaru Yamashita

Kagoshima University: Kagoshima Daigaku

Tomohiro Iwasaki

Shizuoka General Hospital: Shizuoka Kenritsu Sogo Byoin

Chinatsu Tsuchiya

Shizuoka General Hospital: Shizuoka Kenritsu Sogo Byoin

Aki Kubota

Shizuoka General Hospital: Shizuoka Kenritsu Sogo Byoin

Kazuki Hirata

Shizuoka General Hospital: Shizuoka Kenritsu Sogo Byoin

Akinori Murakami

Shizuoka General Hospital: Shizuoka Kenritsu Sogo Byoin

Aya Muramatsu

Shizuoka General Hospital: Shizuoka Kenritsu Sogo Byoin

Kazumori Arai

Shizuoka General Hospital: Shizuoka Kenritsu Sogo Byoin

Makoto Suzuki

Shizuoka General Hospital: Shizuoka Kenritsu Sogo Byoin

Short report

Keywords: Periostin expression, Thyroid tumors, PON, PAC and MIC, immunohistochemical study

Posted Date: February 15th, 2021

DOI: https://doi.org/10.21203/rs.3.rs-202219/v1

License: (c) (i) This work is licensed under a Creative Commons Attribution 4.0 International License.

Read Full License 
1 Periostin expression and its roles in benign and malignant thyroid nodules: An

2 immunohistochemical study of 105 cases

3

$4 \quad{ }^{1}$ Kimihide Kusafuka, D.D.S., Ph.D. (k-kusafuka@i.shizuoka-pho.jp); ${ }^{2}$ Masaru

5 Yamashita, M.D., Ph.D. (yamashita@kufm.kagoshima-u.ac.jp); ${ }^{1}$ Tomohiro Iwasaki,

$6 \quad$ M.T., C.T. (iwasaki.kensou.kensa@gmail.com); ${ }^{1}$ Chinatsu Tsuchiya, M.T., C.T.

7 (tsuchiya.kensou.kensa@gmail.com); ${ }^{1}$ Aki Kubota, M.T.

$8 \quad$ (kubota.kensou.kensa@gmail.com); ${ }^{1}$ Kazuki Hirata, M.T., C.T.

9 (paolo_kazuki@yahoo.co.jp); ${ }^{1}$ Akinori Murakami, M.D. (mrk111111@yahoo.co.jp);

10 19ya Muramatsu, M.D., Ph.D. (aya-muramatsu@i.shizuoka-pho.jp); ${ }^{1}$ Kazumori Arai,

11 M.D., Ph.D. (m-arai@ny.tokai.or.jp); ${ }^{1}$ Makoto Suzuki, M.D., Ph.D. (makoto-

12 suzuki@i.shizuoka-pho.jp)

$13{ }^{1}$ Department of Pathology, Shizuoka General Hospital, Shizuoka, Japan

$14{ }^{2}$ Department of Otorhinolaryngology and Head and Neck Surgery, Kagoshima

15 University, Kagoshima, Japan

17 Correspondence to: Kimihide Kusafuka, D.D.S., Ph.D.

18 Department of Pathology, Shizuoka General Hospital, 
1 4-27-1 Kita-ando, Aoi-ku,

2 Shizuoka City, Shizuoka 420-8527, Japan

3 Tel: +81-54-247-6111; Fax: +81-54-247-6140

4 E-mail: k-kusafuka@i.shizuoka-pho.jp 
1 Abstract: Background. Thyroid tumors are often difficult to histopathologically

2 diagnose, especially follicular adenoma (FA) and follicular carcinoma (FC). Papillary

3 carcinoma (PAC) has several histological subtypes. Periostin (PON), which is a non-

4 collagenous extracellular matrix molecule, is related to tumor invasiveness. We aimed to

5 elucidate the role of PON in thyroid tumors. Method. We collected 105 cases of thyroid

6 nodules, which included cases of adenomatous goiter, FA, microcarcinoma (MIC), PAC,

$7 \quad \mathrm{FC}$, poorly differentiated carcinoma (PDCa), and undifferentiated carcinoma (UCa), and

8 immunohistochemically examined the PON expression patterns of these lesions. Results.

9 PAC and MIC exhibited stromal PON deposition, especially in the solid/sclerosing

10 subtype, whereas FA and FC showed weak deposition on the fibrous capsule. However,

11 the invasive and/or extracapsular regions of microinvasive FC demonstrated quite strong

12 PON expression. Except for it, we could not find any significantly histopathological

13 differences between FA and FC. Although PDCa showed a similar PON expression

14 pattern to PAC, UCa exhibited stromal PON deposition in its invasive portions and

15 cytoplasmic expression in its carcinoma cells. Although there was only one case of UCa,

16 it demonstrated strong PON immunopositivity. PAC and MIC showed similar patterns of

17 stromal PON deposition, especially at the invasive front. Conclusions. PON plays a role

18 in the invasion of thyroid carcinomas, especially PAC and $\mathrm{UCa}$, whereas it acts as a 
1 barrier against the growth of tumor cells in FA and minimally invasive FC.

2

3 Running title: Periostin in thyroid nodules 


\section{Introduction}

2 Papillary carcinoma (PAC) is the most common tumor in the thyroid gland [1]. As

3 for follicular thyroid lesions, it is difficult to differentiate between follicular adenoma

4 (FA) and follicular carcinoma (FC) [2]. As FA and FC, especially the microinvasive

5 type, exhibit similar cellular atypia, the key to their diagnosis is whether they exhibit

6 capsular invasion.

$7 \quad$ Tumors are composed of parenchymal tissue (the tumor cells themselves) and the

8 stroma, which contains the extracellular matrix (ECM) and myofibroblasts (cancer-

9 associated fibroblasts: CAF). The stroma is not only a supportive tissue, but also

10 regulates the migration, differentiation, and/or growth of tumor cells in the tumor

11 microenvironment [3]. Periostin $(\mathrm{PON})$ is a non-collagenous ECM molecule, and it is

12 deposited on the periosteum and periodontal ligaments [4,5]. Recently, PON has been

13 found to be expressed in several cancers, including oral cancer and breast cancer, and to

14 play direct roles in the invasion and migration of tumor cells [6-9]. Moreover, PON-

15 positive cancers have poor prognoses. We examined the expression and localization of

16 PON in thyroid nodules. 
1 We randomly selected 105 cases of thyroid nodules from our institution's pathology

2 files (study period: 2014-2018). They included 10 cases of adenomatous goiter (AG), 13

3 cases of microcarcinoma (MIC), 20 cases of FA, 18 cases of FC, 41 cases of PAC, 2

4 cases of poorly differentiated carcinoma (PDCa), and one case of undifferentiated

5 carcinoma (UCa). As negative controls, the background normal thyroid tissues were

6 selected in matching cases. The clinicopathologic features of the cases using in this

$7 \quad$ study were summarized in Supplemental table 1.

8 We re-diagnosed each case based on hematoxylin and eosin (H\&E) staining and

9 selected specimens that exhibited typical histology. Immunohistochemical staining was

10 performed on $4-\mu m$-thick sections, which were cut from formalin-fixed and paraffin-

11 embedded tissue. Immunostaining was performed according to the relevant

12 manufacturer's instructions, using a Leica BOND MAX automated immunostainer

13 (Leica, Bannockburn, IL, USA) or a Ventana Benchmark GX automatic immunostainer

14 (Roche Tissue Diagnosis, Oro Valley, AZ, USA). The antibodies used in this study are

15 summarized in Table 1 . The tumors were considered to be diffusely positive $(++)$,

16 positive $(++)$, focally positive $(\mathrm{F}+)$, and negative when $\geq 50 \%, 10-49 \%, 1-9 \%$, and $0 \%$

17 of the neoplastic cells were positive, respectively. In the immunohistochemical

18 assessment of PON expression, stromal localization was recorded as S, whereas 
1 as widely invasive FC (WI-FC). The PDCa showed a solid and/or insular pattern. The

cytoplasm localization was recorded as CY. We used Image J (National Institutes of Health, Bethesda, MD, USA) to estimate the percentage of Ki-67-positive tumor cells. 3

\section{Results}

1. Histological findings

All of the MIC were histologically classified as tiny PAC (Figure 1A). There were 0

cases of the encapsulated (cap)/sclerosing ( $\mathrm{scl}$ ) subtype, 5 cases of the non-cap/scl subtype, 6 cases of the cap/non-scl subtype, and 2 case of the non-cap/non-scl subtype.

All of the PAC belonged to the well differentiated type (Figure 1B), and, according to their macroscopic findings, there were 23 cases of the localized/solid subtype, 7 cases of the localized/cystic subtype, and 11 cases of the mixed subtype. The present study did not include any cases of the diffuse sclerosing subtype of PAC. The FA showed dense follicle proliferation and thick fibrous capsules without invasion (Figure 1C). On the other hand, 15 of the 18 cases of FC harbored dense fibrous capsules with extracapsular invasion, which were subclassified into minimally invasive FC (MinI-FC) (Figure 1D).

The remaining 3 cases of FC did not possess fibrous capsules, and these were classified UCa was composed of densely packed proliferating spindle-shaped cells and polygonal 
1 cells, which exhibited marked cellular atypia, and a small focus of the follicular variant

2 of PAC (Figure 1E). The AG did not possess fibrous capsules, but were well-defined.

3 Some areas exhibited dense follicle proliferation, whereas others showed loosely

4 distributed follicles with an edematous stroma (Figure 1F).

6 2. Immunohistochemical analysis of PON expression

$7 \quad$ The immunohistochemical results are summarized in Table 2.

8 The scl subtype of MIC showed moderate positivity for PON, whereas the non-scl

9 subtype did not express PON. In the cap and non-cap subtypes of MIC, PON was

10 localized in the fibrous capsule-like stroma and hyalinized stroma, except in non-scl-

11 type MIC (Figure 2A).

In the localized cystic subtype of PAC, PON was weakly expressed on cyst walls,

13 whereas moderate and strong positivity for PON were seen in the hyaline stroma in the

14 other subtypes of PAC. However, no immunopositivity for PON was observed in the

15 cytoplasm of the cancer cells (Figure 2B). PON was localized around calcified areas or

16 psammoma bodies. The follicular variant of PAC showed scant stromal tissue and less

17 PON expression (Supplemental figure 1). The PAC cases that exhibited direct invasion

18 into the thyroid capsule exhibited stronger immunopositivity for PON than the others. 
FA showed weak PON expression on the fibrous capsule (Figure 2C), which was

2 similar to that seen in MinI-FC. However, WI-FC showed stronger PON expression at

3 the invasive tip than in the inner stroma (Figure 2D).

4 As PDCa exhibited medullary growth, PON was localized in the scant stroma. The

5 UCa demonstrated both stromal and cytoplasmic PON expression (Figure 2E). AG

6 showed very weak or no PON expression (Figure 2F).

7

8 3. Immunohistochemical analysis of other markers

9 PAC and MIC were mainly positive for cytokeratin (CK) 19, whereas AG, FA, and

10 FC were only focally immunopositive for CK19. MIC, PAC, and WI-FC were also

11 positive for galectin-3 (Gal-3) (Figures 3A and C), whereas AG and FA were immunonegative for Gal-3. MinI-FC showed focal immunopositivity for Gal-3. MIC

13 and PAC exhibited diffuse nuclear immunopositivity for cyclin D1 (Figure 3B), whereas

14 FA and AG were negative for cyclin D1. FC, especially the WI type; PDCa; and UCa

15 were partially immunopositive for Hector Battifora mesothelial 1 (HBME-1) and cyclin

16 D1. UCa showed mosaic patterns of immunopositivity for CK19 (Figure 3D) and Ki-67

17 (Figure 3E). PAC and MIC were immunopositive, to varying extents, for HBME-1

18 (Figure 3E) and CK19 (Figure 3F), whereas FA and FC were negative or only focally 
1 positive for HBME-1. AG was immunonegative for HBME-1. FC, especially the WI

2 type; PDCa; and UCa were partially immunopositive for HBME-1 and cyclin D1.

3 The Ki-67 L.I. of AG, MIC, PAC, FA, FC, PDCa, and UCa were $<1 \%, 1-5 \%, 2-9 \%$,

$4 \quad 3 \%, 3 \%, 11 \%$, and $65 \%$, respectively (Figures $3 \mathrm{G}$ and $\mathrm{H}$ ). In pT4a and/or pEx1 (thyroid

5 capsular invasion+) PAC, the Ki-67 L.I. was somewhat higher. The Ki-67 L.I. of FA and

$6 \quad$ FC were similar. UCa had a significantly higher Ki-67 L.I. than the other thyroid

7 tumors/nodules.

8

9 Discussion

10 PON is a glycoprotein, consisting of a $93.3-\mathrm{kDa}$ homodimer, and it was first

11 described as osteoblast-specific factor 2 in $1993[4,10]$. Although PON was initially

12 considered to be related to the adhesion of pre-osteoblasts [4], it also plays important

13 roles in the formation of collagen fibers, cell-cell adhesion, and wound repair [11-14].

14 Moreover, PON has been reported to be associated with invasion capacity and stromal

15 formation in several types of cancer $[6-9,15,16]$. In particular, CAF secrete epidermal

16 growth factor and insulin-like growth factor-1, and those molecules promote tumor

17 growth. Moreover, CAF secrete hepatocyte growth factor/scattering factor, which

18 promotes cell migration [3]. On the other hand, CAF are also involved in the 
1 construction and/or remodeling of the ECM; i.e., they play a role in dissolving types I,

2 III, and V collagen; fibronectin; and laminin, and therefore, promote the migration and

3 invasion of cancer cells [17]. PON have been reported to play these roles in non-small

4 cell lung cancer, oral cancer, and liver cancer $[6-9,15,16]$. In numerous types of cancer,

5 cancer cells themselves have been reported to secrete PON, but stromal CAF also

6 secrete PON, which promotes the migration and/or invasion of cancer cells $[18,19]$.

7 Ratajczak-Wielgomas et al. reported that benign lesions of the breast showed no stromal

8 PON deposition, but PON was deposited around the malignant mammary ducts in

9 ductal carcinoma in situ, and it was more widely deposited in the cancer stroma in

10 invasive ductal carcinoma of the breast, especially in cases showing high-grade

11 histological atypia [19]. The PON secreted by CAF not only plays a role in tumor cell

12 migration and invasion, but is also associated with tumor cell differentiation and high-

13 grade malignancy via epithelial-mesenchymal transition $[19,20]$.

14 In the present study, we examined the expression and localization of PON in thyroid

15 tumors. Cytoplasmic PON localization was not observed in our series, except in UCa.

16 This may have been because the antibody used in our study recognized a different

17 epitope to those used in previous studies. Alternatively, it could have been due to the

18 slow growth of thyroid tumors (even malignant tumors), except for UCa. 
1 As AG is not associated with CAF recruitment and is a hyperplastic lesion, AG showed

2 only a little PON expression. On the other hand, PON was weakly expressed on the

3 fibrous (pseudo-)capsules in the tumors with fibrous capsules (i.e., cap-MIC, FA, and

4 the localized/cystic type of PAC), whereas PON was expressed in the hyaline stroma in

5 the stroma-rich tumors. In UCa, which is a rapidly growing tumor, PON was not

6 strongly expressed in the stroma or the tumor cytoplasm. Therefore, we consider that

$7 \quad$ PON is not related to the growth capacity of thyroid tumors.

8 Two reverse transcription polymerase chain reaction (RT-PCR)-based studies of PON

9 expression in PTC have been reported $[21,22]$. They indicated that PON mRNA

10 expression was higher in tumor tissues than in normal tissues. Bai et al. detected high

11 PON mRNA expression in cases of PAC involving extracapsular invasion, and they

12 reported that in PAC PON expression was higher at the invasive front and was related to

13 loss of polarity in cancer cells [21]. However, the localization of PON in PAC remained

14 unknown. In the present study, PON was deposited in the fibrous stroma in PAC, and

15 stronger deposition was seen in the cases involving invasion into the thyroid capsule.

16 There are 5 wild-type isoforms of PON. Using RT-PCR and direct DNA sequencing,

17 Bai et al. found three new isoforms of PON mRNA (variations were seen on the C-

18 terminal side) in PAC [22], but their clinicopathological significance remains unclear. In 
1 urothelial carcinoma, it was reported that some cases expressed variant I (loss of exons

2 XVII, XVIII, and XXI) whereas others expressed variant II (loss of exons XVII and

$3 \mathrm{XXI}$ [23], and variant I was found to be associated with in vitro invasiveness and in

4 vivo metastatic capacity. Similar association might exist in PAC.

5 In this study, we also examined thyroid tumors other than PAC, and PON deposition

6 was seen on the thick fibrous capsule in FA and MinI-FC. However, MIC showed

7 stronger PON deposition than FA and MinI-FC. These results suggest that PON plays a

8 role in capsular formation and/or acts as barrier against tumor extension or invasion.

9 We immunohistochemically examined the expression of HMBE-1 and Gal-3. As

10 reported previously, most cases of PAC were positive for both markers, whereas

11 approximately half of cases of FA and 70\% cases of MinI-FC showed partial expression

12 of these PAC markers [24,25]. Immunohistochemically examining the expression of

13 both HMBE-1 and Gal-3 is considered to be useful for the differential diagnosis of

14 thyroid tumors. Recently, strong expression of cyclin D1 in MIC (tiny PAC type) was

15 reported to be positively correlated with extracapsular invasion [26], and the present

16 study showed that all cases of MIC belonged to the tiny PAC type. Twelve (92\%) of the

1713 MIC cases and 38 (93\%) of 41 PAC cases were positive for cyclin D1, and cyclin D1

18 positivity was not related to extracapsular invasion or lymph node metastasis. 


\section{Conclusions}

3 In conclusion, stromal PON deposition in PAC was found to be related to stromal

4 formation and invasion, whereas PON deposition on the fibrous capsules of FA or MinI-

$5 \quad$ FC may function as a barrier against tumor extension. PON plays different roles in each

6 histological type of thyroid tumor.

7

9 List of abbreviations:

10 PAC, papillary carcinoma; FC, follicular carcinoma; FA, follicular adenoma; ECM,

11 extracellular matrix; CAF, cancer-associated fibroblasts; PON, periostin; PDCa, poorly

12 differentiated carcinoma; UCa, undifferentiated carcinoma; MIC, microcarcinoma; H\&E,

13 hematoxylin and eosin; cap, encapsulated; scl, sclerosing; AG, adenomatous goiter; MinI-

14 FC, minimally invasive follicular carcinoma; WI-FC, widely invasive FC; CK,

15 cytokeratin; HBME-1, Hector Battifora mesothelial-1; Gal-3, galectin-3; L.I., labeling

16 index; RT-PCR, reverse transcription polymerase chain reaction

\section{Declarations}


1 Ethical approval and consent to participate

2 This study was approved by the institutional review board of Shizuoka General

3 Hospital (SGHIRB\#2019007). All subjects signed informed consent forms before

4 participating.

5

6 Consent for publication

$7 \quad$ All subjects signed informed consent forms before participating.

8

$9 \quad$ Availability of data and materials

10 The datasets used and analyzed during the current study are available from the

11 corresponding author on reasonable request.

13 Competing interests

14 The authors declare that they have no competing interests relating to this study.

16 Funds

17 This study was supported in part by a Grant-in-Aid from the Medical Research Support

18 Project of Shizuoka Prefectural Hospital Organization in 2019 (to KK). 
1 Authors' contributions

2 KK designed and drafted the manuscript, and KK, AMurakami, AMuramatsu, KA,

3 and MS made the histopathological diagnoses and analyzed the immunohistochemical

4 results. TI, CT, AK, and $\mathrm{KH}$ performed the excellent immunohistochemistry. MY

5 provided the clinical data. MS supervised the manuscript. All of the authors have read

6 and approved the final manuscript.

7

8 Acknowledgements

9 The authors thank Mr. Naofumi Ishikawa, Ms. Yoko Yamazaki, Mr. Kensuke

10 Simazaki, Mr. Koji Takahashi, Ms. Nobuyo Tsujino, and Mr. Yohei Saguchi (medical

11 staff at the Department of Pathology, Shizuoka General Hospital, Shizuoka, Japan) for

12 their excellent technological co-operation. We are also grateful to Ms. Mamiko Uemura

13 (the secretary at the Department of Pathology, Shizuoka General Hospital) for her help

14 with the manuscript preparation. 


\section{$1 \quad$ References}

2 1. Rosai J, Albores Saavedra J, Asioki S, Bolach ZW, Bogdova T, Chen H, et al.

3 Papillary carcinoma (eds.) Lloyd RV, Osamura RY, Kloppel G, Rosai J. WHO

4 Classification of Tumours of Endocrine Organs. 4th edition IARC Press, Lyon, France.

5 pp81-91.

6 2. LiVolsi V, Abdulkader Nalib I, Baloch ZW, Bartolazzi A, Chan JKC, DeLellis RA, et

7 al. Follicular thyroidal carcinoma (eds) Lloyd RV, Osamura RY, Kloppel G, Rosai J.

8 WHO Classification of Tumours of Endocrine Organs. 4th edition IARC Press, Lyon,

$9 \quad$ France. pp92-5.

10 3. Santi A, Kugeratski FG, Zanivan S. Cancer associated fibroblasts: The architects of

11 stroma remodeling. Proteomics 2018: doi: 10.1002/pmic.201700167,

12 4. Horiuchi K, Amizuka N, Takeshita S, Takamatsu H, Katsuura M, Ozawa H, et al.

13 Identification and characterization of a novel protein, periostin, with restricted

14 expression to periosteum and periodontal ligament and increased expression by

15 transforming growth factor beta. J Bone Miner Res 1999;14:1239-49.

16 5. Kudo A. Periostin in bone biology. Adv Exp Med Biol 2019;1132:43-7.

17 6. Nuzzo PV, Rubagotti A, Zinoli L, Salvi S, Boccardo S, Boccardo F. The prognostic

18 value of stromal and epithelial periostin expression in human breast cancer: Correlation 
1 with clinical pathological features and mortality outcome, BMC Cancer 2016; doi:

2 10.1186/s12885-016-2139-y.

3 7. Shao R, Bao B, Bai X, Blanchette C, Anderson RM, Dang T, et al. Acquired expression

4 of periostin by human breast cancers promotes tumor angiogenesis through up-

5 regulation of vascular endothelial growth factor receptor 2 expression. Mol Cell Biol

$6 \quad 2004 ; 24: 3992-4003$.

7 8. Siriwardena BS, Kudo Y, Ogawa I, Kitagawa M, Kitajima S, Hatano H, et al. Periostin

8 is frequently overexpressed and enhances invasion and angiogenesis in oral cancer. $\mathrm{Br} \mathrm{J}$

9 Cancer 2006;95:1396-403.

10 9. Jang SY, Park SY, Lee HW, Choi Y-K, Park K-G, Yoon GS, et al. The combination of

11 periostin overexpression and microvascular invasion in related to a poor prognosis for

12 hepatocellular carcinoma. Gut Liver 2016;10:948-54.

13 10. Takeshita S, Kikuno R, Tezuka K, Amann E. Osteoblast-specific factor 2: Cloning

14 of a putative bone adhesion protein with homology with insert protein fasciclin I.

15 Biochem J 1993; 294(Pt1): 271-8. 
1 11. Nunomura S, Nanri Y, Okawa M, Arima K, Mitamura Y, Yoshihara T, et al.

2 Constitutive overexpression of periostin delays wound healing in mouse skin. Wound

$3 \quad$ Repair Regen 2018;26:6-15.

4 12. Cobo T, Viloria CG, Solares L, Fontanil T, Gonzalez-Chamorro E, De Carlos F, et

5 al. Role of periostin in adhesion and migration of bone remodeling cells. PLoS One

$6 \quad$ 2016;11:e0147837. doi: 10.1371/journal.pone.0147837.

7 13. Zhao S, Wu H, Xia W, Chen X, Zhu S, Zhang S, et al. Periostin expression is

8 upregulated and associated with myocardial fibrosis in human failing hearts. J

9 Cardiol 2014;63:373-8.

10 14. Braun N, Sen K, Alscher MD, Fritz P, Kimmel M, Morelle J, et al. Periostin: A

11 matricellular protein involved in peritoneal injury during peritoneal dialysis. Perit

12 Dial Int 2013; 33: 515-28.

15. Nuzzo PV, Buzzatti G, Ricci F, Rubagotti A, Argellati F, Zinoli L, et al. A novel

14 prognostic and therapeutic target for genitourinary cancer? Clin Genitourin Cancer 2014;12:301-11. 
1 16. Takanami I, Abiko T, Koizumi S. Expression of periostin in patients with non-

2 small-cell lung cancer: Correlation with angiogenesis and lymphangiogenesis. Int $\mathbf{J}$

3 Biol Markers 2008;23:182-186.

4 17. Nissen NI, Karsdal M, Willumsen N. Collagens and cancer associated fibroblasts in

5 the reactive stroma and its relation to cancer biology. J Exp Clin Cancer Res 2019;

6 doi: 10.1186/s13046-019-1110-6.

7 18. Oh HJ, Bae JM, Wen XY, Cho NY, Kim JH, Kang GH. Overexpression of POSTN

8 in tumor stroma is a poor prognostic indicator of colorectal cancer. J Pathol Trans

$9 \quad$ Med 2017;51(3):306-13.

10 19. Ratajczak-Wielgomas K, Grzegrzolka J, Piotrowska A, Gomulkiewicz A,

11 Witkiewicz W, Dziegial P. Periostin expression in cancer-associated fibroblasts of

12 invasive ductal breast carcinoma. Oncol Rep 2016;36:2745-54.

13 20. Kikuchi Y, Kunita A, Iwata C, Komura D, Nishiyama T, Shimazu K, et al. The

14 niche component periostin is produced by cancer-associated fibroblasts, supporting

15 growth of gastric cancer through ERK activation. Am J Pathol 2014;184:859-70. 
1 21. Bai Y, Kakudo K, Nakamura M, Ozaki T, Li Y, Liu Z, et al. Loss of cellular

2 polarity/cohesiveness in the invasive front of papillary thyroid carcinoma and

3 periostin expression. Cancer Let 2009;281:188-95.

4 22. Bai Y, Nakamura M, Zhou G, Li Y, Liu Z, Ozaki T, et al. Novel isoforms of

5 periostin expressed in the human thyroid. J Clin Med 2010; 1: 13-20.

6 23. Kim CJ, Isono T, Tambe Y, Chano T, Okabe H, Okada Y, et al. Role of alternative

$7 \quad$ splicing of periostin in human bladder carcinogenesis. Int J Oncol 2008;32:161-9.

8 24. Saleh HA, Jin B, Barnwell J, Alzohaili O. Utility of immunohistochemical markers

9 in differentiating benign from malignant follicular-derived thyroid nodules. Diag

10 Pathol 2010; doi: 10.1186/1746-1596-5-9.

11 25. Prasad ML, Pellegata NS, Huang Y, Nagaraja HN, de la Chapelle A, Kloos RT.

12 Galectin-3, fibronectin-1, CITED-1, HBME-1 and cytokeratin-19

13 immunohistochemistry is useful for the differential diagnosis of the thyroid tumors.

$14 \quad$ Mod Pathol 2005;18:48-57. 
1 26. Juan C-K, Kang Y-G, Bae J-S, Lim D-J, Choi Y-J, Lee K-Y. Unique patterns of

2 tumor growth related with the risk of lymph node metastasis in papillary thyroid

3 carcinoma. Mod Pathol 2010; 3:1201-8. 


\section{$1 \quad$ Figure legends}

2 Figure 1. Histology of the thyroid nodules

3 (A) Microcarcinoma (H\&E). The nuclei of the tumor cells showed a ground glass-like

4 appearance (inset), and the tumor cells were surrounded by a sclerotic stroma. Papillary

5 carcinoma (H\&E). Tumor cells, whose nuclei exhibited a ground glass-like appearance,

6 formed irregular papillary structures. (C) Follicular adenoma (H\&E). Follicles

7 composed of tumor cells that exhibited mild nuclear atypia were seen, but no capsular

8 invasion was observed (fc, fibrous capsule). (D) Minimally invasive follicular

9 carcinoma (H\&E). Tumor cells that exhibited mild atypia had invaded the fibrous

10 capsule (fc, fibrous capsule; arrows, capsular invasion). (E) Undifferentiated carcinoma

11 (H\&E). Polymorphous atypical short spindle-shaped cells that exhibited loose cell-cell

12 adherence were arranged in fascicular structures or diffusely distributed. Adenomatous

13 goiter (H\&E). Hyperplastic follicular cells without atypia were seen. The follicles

14 contained colloid.

16 Figure 2. Results of immunohistochemical periostin (PON) staining for each thyroid

17 nodule 
1 (A) Microcarcinoma. Immunopositivity for PON was observed in the sclerotic stroma.

2 (B) Papillary carcinoma. Moderate to strong immunopositivity for PON was observed

3 in the sclerotic stroma at the invasive front (arrows). (C) Follicular adenoma. Weak

4 immunopositivity for PON was seen in the fibrous capsule (fc), together with some

5 calcification (cal). (D) Widely invasive follicular carcinoma. Diffuse immunopositivity

6 for PON was seen in the sclerotic stroma in the invasive regions. (E) Adenomatous

7 goiter. Very weak signals for PON were seen in the pseudo-capsule (ps-fc) around the

8 nodule in adenomatous goiter. (F) Undifferentiated carcinoma. Strong immunopositivity

9 for PON was diffusely observed in the stroma around the cancer cells. (F, inset) PON-

10 immunoreactivity was also seen in the cytoplasm of the cancer cells.

12 Figure 3. Immunohistochemical staining results for other thyroid tumor markers

13 In microcarcinoma, the cancer cells exhibited strong positivity for galectin-3 (Gal-3)

14 (A) and nuclear positivity for cyclin D1 (B). In papillary carcinoma, the cytoplasm of

15 the cancer cells was diffusely positive for Gal-3 (C). Undifferentiated carcinoma

16 showed cytoplasmic immunopositivity for Gal-3 with a mosaic-like pattern (D). In

17 papillary carcinoma, the luminal side of the papillary structures was positive for Hector 
1 Battifora mesothelial 1 (HBME-1) (E), and the tumor cells were diffusely positive for

2 cytokeratin (CK) 19 (F). In undifferentiated carcinoma, the cancer cells were diffusely

3 positive for Ki-67 (G), whereas in papillary carcinoma the tumor cells were sporadically

4 positive for $\mathrm{Ki}-67(\mathrm{H})$. 


\section{Figures}

\section{Figure 1}
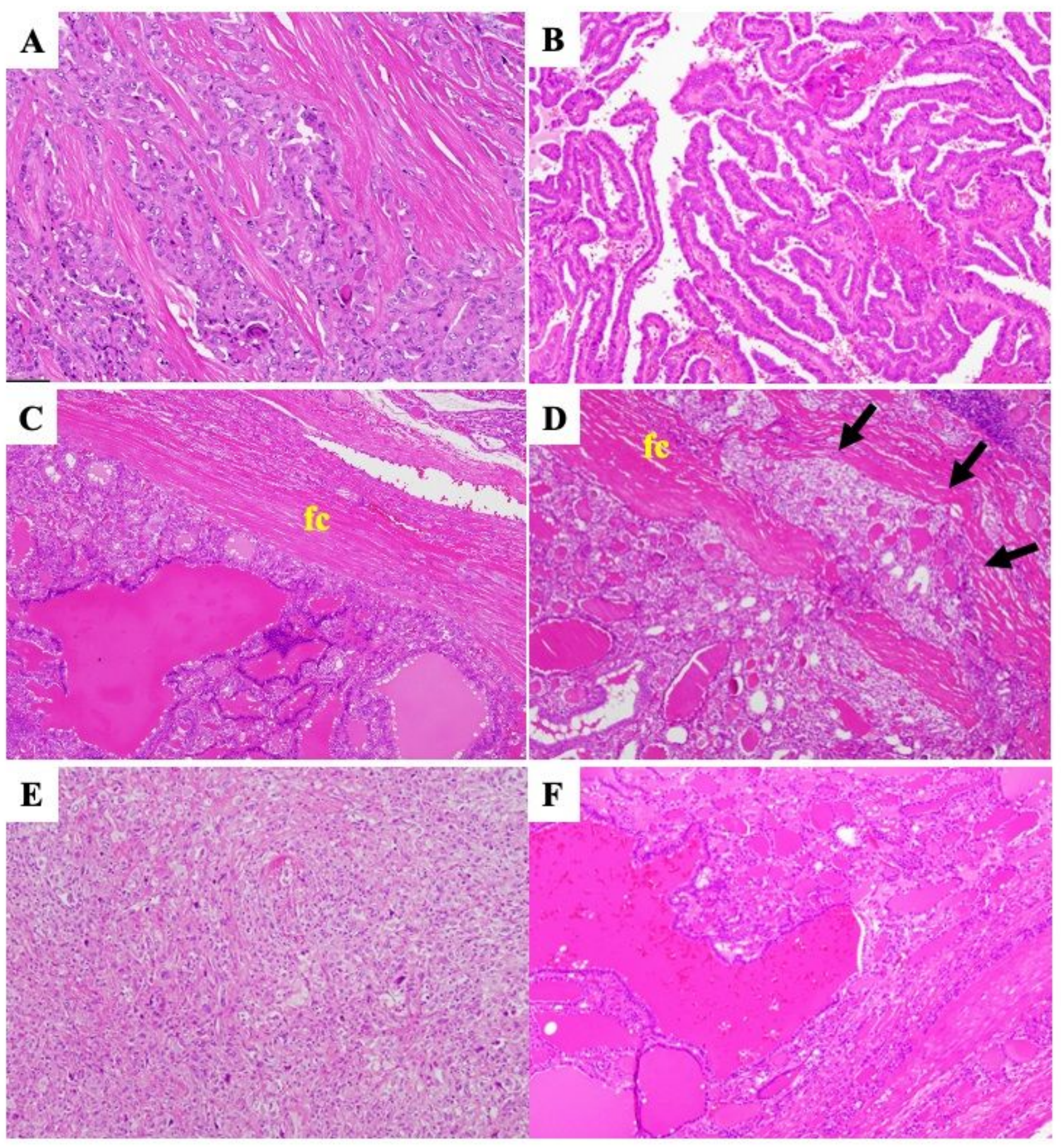

\section{Figure 1}

Histology of the thyroid nodules (A) Microcarcinoma (H\&E). The nuclei of the tumor cells showed a ground glass-like appearance (inset), and the tumor cells were surrounded by a sclerotic stroma. Papillary carcinoma (H\&E). Tumor cells, whose nuclei exhibited a ground glass-like appearance, formed irregular 
papillary structures. (C) Follicular adenoma (H\&E). Follicles composed of tumor cells that exhibited mild nuclear atypia were seen, but no capsular invasion was observed (fc, fibrous capsule). (D) Minimally invasive follicular carcinoma (H\&E). Tumor cells that exhibited mild atypia had invaded the fibrous capsule (fc, fibrous capsule; arrows, capsular invasion). (E) Undifferentiated carcinoma (H\&E). Polymorphous atypical short spindle-shaped cells that exhibited loose cell-cell adherence were arranged in fascicular structures or diffusely distributed. Adenomatous goiter (H\&E). Hyperplastic follicular cells without atypia were seen. The follicles contained colloid.

\section{Figure 2}

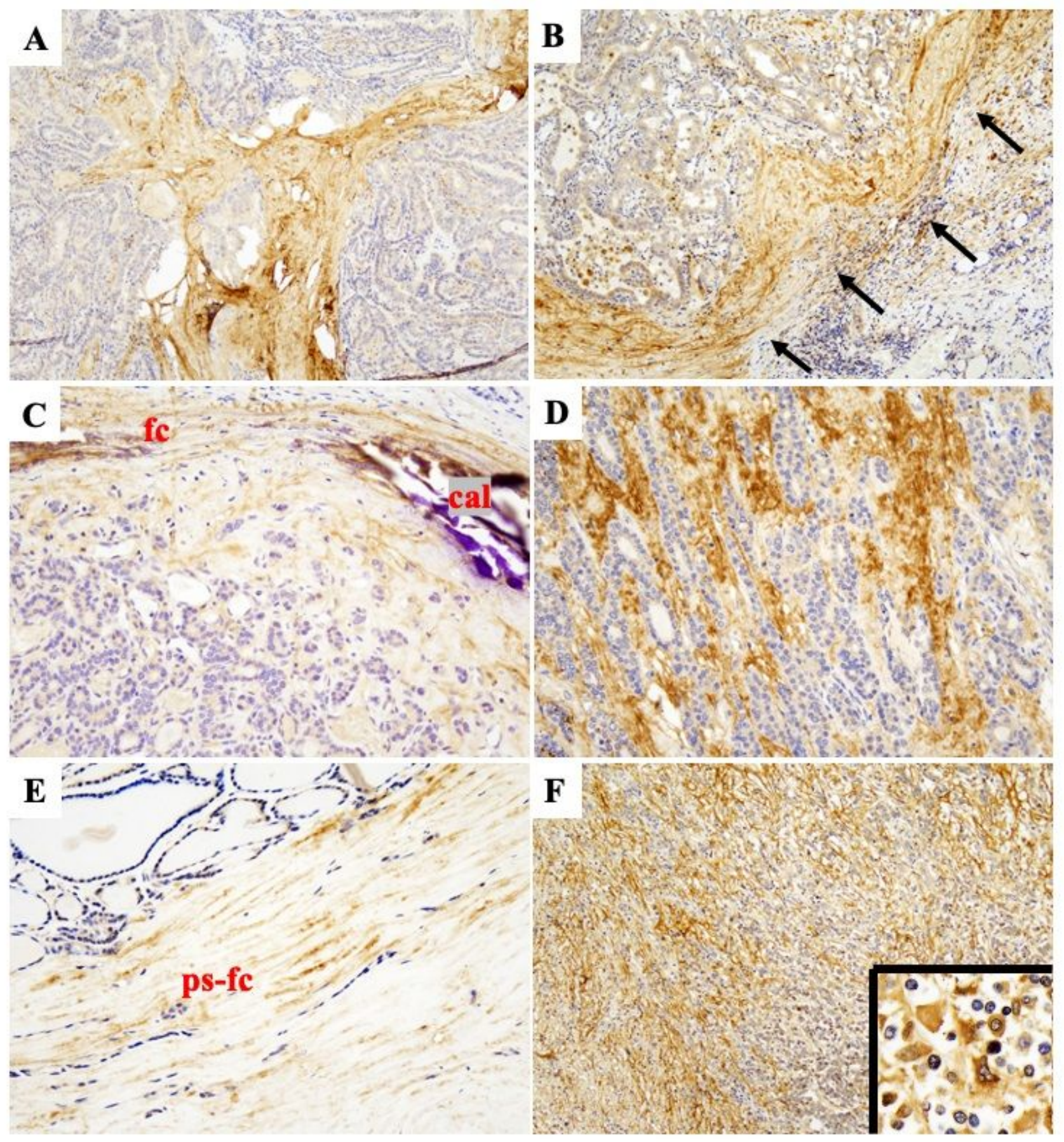




\section{Figure 2}

Results of immunohistochemical periostin (PON) staining for each thyroid nodule (A) Microcarcinoma. Immunopositivity for PON was observed in the sclerotic stroma. (B) Papillary carcinoma. Moderate to strong immunopositivity for PON was observed in the sclerotic stroma at the invasive front (arrows). (C) Follicular adenoma. Weak immunopositivity for PON was seen in the fibrous capsule ( $\mathrm{fc}$ ), together with some calcification (cal). (D) Widely invasive follicular carcinoma. Diffuse immunopositivity for PON was seen in the sclerotic stroma in the invasive regions. (E) Adenomatous goiter. Very weak signals for PON were seen in the pseudo-capsule (ps-fc) around the nodule in adenomatous goiter. (F) Undifferentiated carcinoma. Strong immunopositivity for PON was diffusely observed in the stroma around the cancer cells. (F, inset) PON-immunoreactivity was also seen in the cytoplasm of the cancer cells. 


\section{Figure 3}
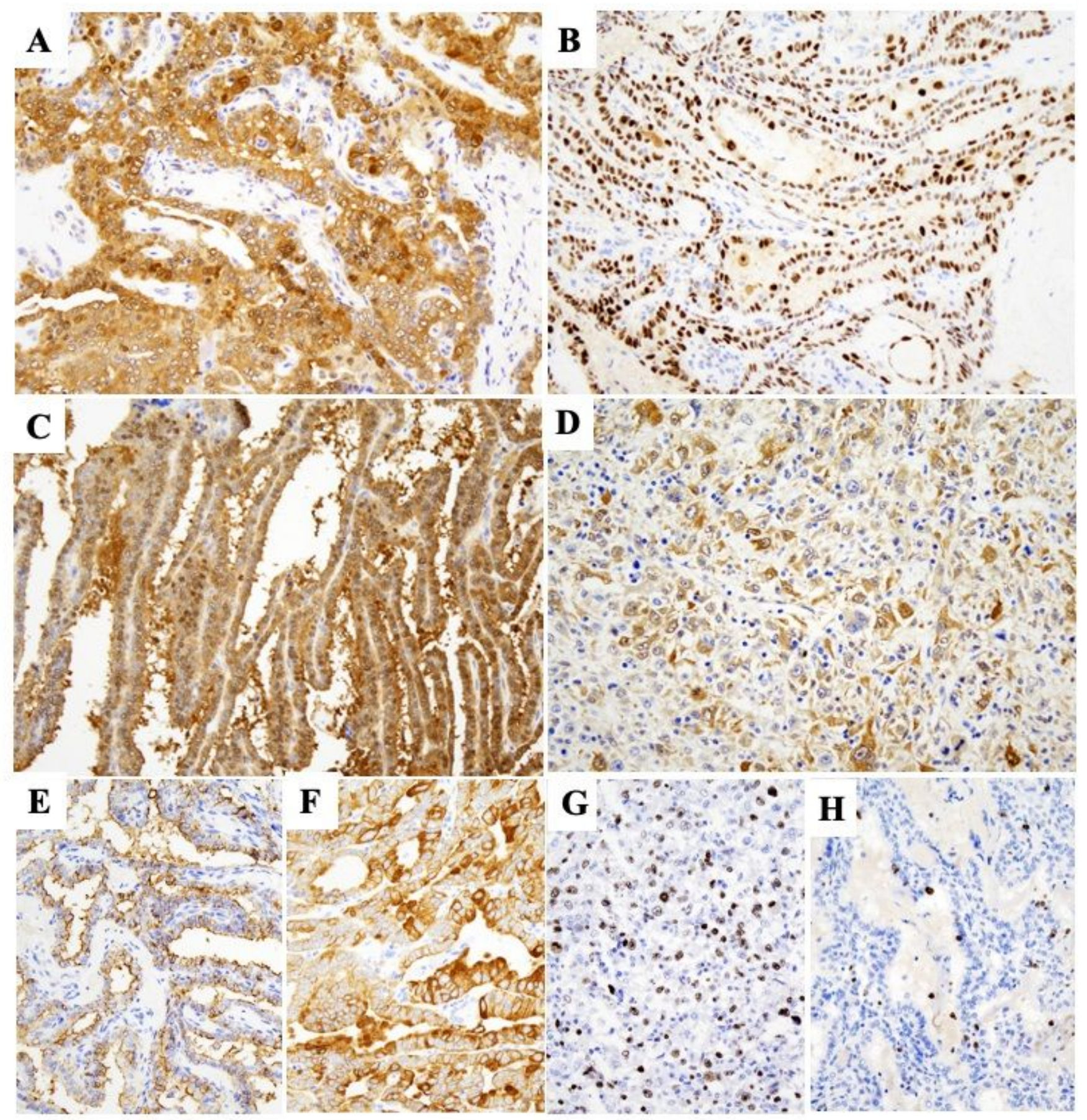

\section{Figure 3}

Immunohistochemical staining results for other thyroid tumor markers In microcarcinoma, the cancer cells exhibited strong positivity for galectin-3 (Gal-3) (A) and nuclear positivity for cyclin D1 (B). In papillary carcinoma, the cytoplasm of the cancer cells was diffusely positive for Gal-3 (C). Undifferentiated carcinoma showed cytoplasmic immunopositivity for Gal-3 with a mosaic-like pattern (D). In papillary carcinoma, the luminal side of the papillary structures was positive for Hector Battifora 
mesothelial 1 (HBME-1) (E), and the tumor cells were diffusely positive for cytokeratin (CK) 19 (F). In undifferentiated carcinoma, the cancer cells were diffusely positive for Ki-67 (G), whereas in papillary carcinoma the tumor cells were sporadically positive for Ki-67 (H).

\section{Supplementary Files}

This is a list of supplementary files associated with this preprint. Click to download.

- PONthyroidSuppl.pdf

- SuppleFig1.tiff 